\title{
Shifting the attention from devices to treatment: the lesson from IABP-SHOCK II and other trials in cardiogenic shock
}

\author{
Francesco Saia \\ Cardiology Unit, Cardio-Thoracic-Vascular Department, University Hospital of Bologna, Policlinico S. Orsola-Malpighi, Bologna, Italy \\ Correspondence to: Francesco Saia, MD, PhD, FESC. Cardiology Unit, Cardio-thoracic-vascular Department, University Hospital of Bologna, \\ Policlinico S.Orsola-Malpighi (Pav. 23), Via Massarenti, 940138 Bologna, Italy. Email: francescosaia@hotmail.com. \\ Provenance: This is an invited article commissioned by the Section Editor Hui-Ping Zhang (Department of Cardiology, Beijing Hospital, the Fifth \\ Affiliated Hospital of Peking University, Beijing, China). \\ Comment on: Thiele H, Zeymer U, Thelemann N, et al. Intraaortic Balloon Pump in Cardiogenic Shock Complicating Acute Myocardial Infarction: \\ Long-Term 6-Year Outcome of the Randomized IABP-SHOCK II Trial. Circulation 2018;139:395-403.
}

Submitted Sep 23, 2019. Accepted for publication Oct 20, 2019.

doi: $10.21037 /$ jtd.2019.10.66

View this article at: http://dx.doi.org/10.21037/jtd.2019.10.66

"Evidence based medicine is the conscientious, explicit, and judicious use of current best evidence in making decisions about the care of individual patients. The practice of evidence based medicine means integrating individual clinical expertise with the best available external clinical evidence from systematic research" Sackett et al., BMJ 1996 (1).

The 2 sentences above summarize the basic essence of evidence-based medicine (EBM). However, as the devil hides in the details, the concept of integrating best evidence with individual clinical expertise harbors the seed of a conflict not always easy to resolve in clinical practice. One of the best examples of the conflict between EBM and clinical practice is the use of mechanical circulatory support (MCS) devices in patients with cardiogenic shock. As clinicians, we recognize that cardiogenic shock is an extremely serious, rapidly evolving condition that sometimes benefits from the use of devices capable of supporting the circulation while coronary revascularization is performed, or that are able to bridge the patient to the next level of care: upgrade of left ventricular assist devices, cardiac surgery for mechanical complications or heart transplant for terminal heart failure. At the same time, as scientists, we must acknowledge the virtual lack of evidence supporting this belief.

Intraaortic balloon pump (IABP) is the most widely used MCS device; it allows augmentation of the diastolic blood pressure, improvement of coronary perfusion and reduction of myocardial oxygen consumption, with a small but significant effect on cardiac output. Importantly, it relies on the existence of a spontaneous circulation and cannot be applied to patients without intrinsic heart action. Until recently, the vast majority of interventional cardiologists, including myself, would have been ready to claim that the IABP was effective beyond any reasonable doubt in improving the prognosis of patients with acute myocardial infarction and cardiogenic shock (AMICS). The argument of the parachute was often used to support the thesis that no formal testing was needed for this postulate (2). Over the years, this seemingly unshakable certainty has been progressively eroded by a series of consistently negative data coming from the randomized IABP-SHOCK II trial, comparing IABP versus control in patients with AMICS undergoing early revascularization (3). The direct consequences of the IABP-SHOCK II trial were: (I) downgrading of routine use of IABP in AMICS to a class III recommendation, level of evidence B, in the European Society of Cardiology guidelines (4); (II) strong reduction of IABP use in clinical practice $(5,6)$; (III) progressive increase in use of different, more powerful, MCS devices like the Impella (Abiomed, Danvers, MA, USA), the Tandem Heart (Cardiac Assist Inc, Pittsburgh, PA, USA), and veno-arterial extracorporeal membrane oxygenation (VA-ECMO).

The last chapter of the IABP-SHOCK II study was the recently published paper reporting the 6-year results (7). After the neutral short-term results of IABP $v s$. control in the primary endpoint analysis, the main rationale to look at the long-term follow-up was that even if a difference 
could not be demonstrated early, perhaps a better early management could be associated with positive effects that manifest only over time. That was the case, for example, of the SHOCK trial, upon which is based the class I recommendation for early revascularization in AMICS (8). To support this hypothesis, in a previous study of IABP in elective high-risk percutaneous coronary intervention (PCI), IABP actually showed no benefit at short-term follow-up but a strong trend for mortality reduction at 5 -year (9). Mirroring the previously reported findings at 30-day and 12-month, however, in the IABP-SHOCK II long-term follow-up there was no significant difference between groups in terms of mortality and quality of life. An important additional finding was that, despite modern medical treatment and state-of-the-art revascularization, twothird of the patients had died at 6 years (7).

Is this the end of the IABP life in this setting? Should we immediately shift to different MCS devices? The answers are not straightforward and source of passionate controversy. For sure, EBM does not support routine use of IABP in AMICS. Disappointingly, there is also no clue about potential utility in any specific subgroup analyzed. According to guidelines, a niche utilization for IABP could be stabilization of patients with a mechanical complication of acute myocardial infarction (4). Before addressing the subgroup issue, let's focus on the second question. There is a solid rationale for use of other and more powerful MCS in AMICS. However, whilst preliminary data for other MCS devices were promising, data from randomized trials powered for clinical outcomes are either lacking or did not support superiority over IABP. The Impella provides better support and a higher cardiac index in comparison with IABP (10). However, with the important limitation of small sample size, the IMPRESS-in-Severe-SHOCK trial showed similar 30-day mortality and increased bleeding in patients treated with the Impella CP (maximum output around 3.7 L/min) as compared with IABP (11). Similar findings came from a matched-pair analysis between Impella and IABP (12) and from retrospective studies (13). Somewhat relevant for the interpretation of the IMPRESS-in-shock trial is the analysis of patient population: $92 \%$ of enrolled patients had been resuscitated from cardiac arrest and almost one-half had time to ROSC longer than $20 \mathrm{~min}$. As a result, $46 \%$ of patients died due to anoxic brain damage vs. $29 \%$ with refractory shock or multiorgan failure (11). In a similar scenario, any potential advantage on cardiac support is likely diluted by the overwhelming impact of adverse neurologic conditions. The evidence is not stronger nor better for
ECMO. It can provide full circulatory support to both the left and the right ventricle and several observational studies reported excellent results (3), but rigorous evaluation is lacking. Further, if surgical insertion was burdened by many complications, percutaneous insertion of VA-ECMO represents an important advancement but the increase in $\mathrm{LV}$ afterload is an important issue and $\mathrm{LV}$ venting is often necessary. Randomized trials with clinical endpoints for ECMO and Impella in AMICS are ongoing (3).

A potentially relevant advancement in management of AMICS came recently from the National Cardiogenic Shock Initiative. Using a standard protocol emphasizing invasive hemodynamic monitoring and early initiation of MCS ( $>90 \%$ Impella) before PCI, this multicenter single-arm registry reported an unprecedented $72 \%$ survival to discharge (14). Additional study finding was the identification of creatinine $\geq 2$, lactate $>4$, cardiac power output $(\mathrm{CPO})<0.6 \mathrm{~W}$, and age $\geq 70$ years as very powerful predictors of mortality.

What conclusions are possible from available data (Table 1)? Remembering that EBM "de-emphasizes intuition, unsystematic clinical experience, and pathophysiologic rationale as sufficient grounds for clinical decision making" (15), we must accept a very marginal role for IABP in AMICS and ask for a rigorous evaluation of other MCS. However, as clinicians, it is our duty to provide patients with the best possible treatment according to our experience and expertise, and sometimes MCS represent an extraordinary ally in the deadly battle against AMICS. While waiting for more data and randomized trials to be completed, the best way to reconcile evidence and experience seems development of protocols that shift the attention from a devices-centered to a patient-centered pathway of care. Selective use of MCS must be in the therapeutic algorithm but it should be guided by pre-defined and measurable parameters. Beyond hemodynamic status (and CPO), age and comorbidity should play a major role: younger patients without severe neurologic compromise, no severe peripheral artery disease and without end-stage disease of other organs could represent the best candidates for MCS.

The next steps will be filling present knowledge gaps including:

(I) Which patient's subgroup do actually benefit from MCS.

(II) Should we start MCS in a progressive fashion (e.g., stepwise escalation from IABP to Impella to ECMO) or start immediately with one of the more powerful devices? In this second scenario, Impella might represent the best choice because it combines 
Table 1 Key messages

There is the urgent need to reduce mortality in AMICS

- Despite state-of-the-art revascularization mortality of AMICS remains in the range of $40-50 \%$ at 30 -day and up to $70 \%$ at 6 -year

IABP should not been used routinely because it does not improve prognosis

o Should be considered when there are mechanical complications of $\mathrm{Ml}$ as underlying causes of cardiogenic shock

- Very selective use could be considered as a support to allow appropriate revascularization, bridge to recover or bridge to other MCS in early stage AMICS

More powerful MCS devices improve circulatory support and surrogate endpoints but in head-to-head comparisons vs. IABP failed to demonstrate clinical superiority and are associated with higher rates of complications

- Their safety and efficacy must be tested in rigorous and adequately powered RCTs

- Before more evidence is available, use of MCS should be integrated within a "best practice", well defined and patient-centered protocol of care for AMICS

Methodological considerations for clinical research

- Need to apply same definitions of cardiogenic shock and its sub-categories (e.g., SCAl classification) across different trials

- Patients with undefined neurologic status should either not be enrolled in MCS trials or separately tested

- Clearly define if there are situations where formal testing can be omitted (MCS parachute-like or end-of-life/futility conditions)

o Test different strategies beyond single devices

AMICS, acute myocardial infarction with cardiogenic shock; IABP, intra-aortic balloon pump; MCS, mechanic cardiac support; RCT, randomized controlled trial.

the advantage of powerful ventricular assistance, is less invasive than ECMO and efficiently unload the left ventricle without the need of an additional venting device.

(III) Best timing for MCS: before or after PCI. The latter hypothesis is aimed at avoiding un-necessary use of MCS in patients that will likely start recovering immediately after PCI. The former, supported by the National Cardiogenic Shock Initiative, is based on the rationale that early LV unload could be beneficial and help best coronary disease treatment.

A strategic help in this scenario comes from the SCAI clinical expert consensus statement on the classification of cardiogenic shock, which may help in predicting prognosis, designing therapeutic algorithm and standardize future clinical research (16).

\section{Acknowledgments}

None.

\section{Footnotes}

Conflicts of Interest: F Saia reports receiving consulting fees from Abbott Vascular, Eli Lilly, Astra Zeneca, St. Jude Medical, Edwards, Medtronic, Amgen, Bayer and speaker's fees from Abbott Vascular, Eli Lilly, Astra Zeneca, St. Jude Medical, Terumo, Biosensors, Edwards, Boston Scientific.

Ethical Statement: The author is accountable for all aspects of the work in ensuring that questions related to the accuracy or integrity of any part of the work are appropriately investigated and resolved.

\section{References}

1. Sackett DL, Rosenberg WM, Gray JA, et al. Evidence based medicine: what it is and what it isn't. BMJ 1996;312:71-2.

2. Smith GC, Pell JP. Parachute use to prevent death and major trauma related to gravitational challenge: systematic review of randomised controlled trials. BMJ 2003;327:1459-61.

3. Thiele H, Ohman EM, de Waha-Thiele S, et al. Management of cardiogenic shock complicating myocardial infarction: an update 2019. Eur Heart J 2019;40:2671-83.

4. Ibanez B, James S, Agewall S, et al. 2017 ESC Guidelines 
for the management of acute myocardial infarction in patients presenting with ST-segment elevation: The Task Force for the management of acute myocardial infarction in patients presenting with ST-segment elevation of the European Society of Cardiology (ESC). Eur Heart J 2018;39:119-77.

5. Shah M, Patnaik S, Patel B, et al. Trends in mechanical circulatory support use and hospital mortality among patients with acute myocardial infarction and noninfarction related cardiogenic shock in the United States. Clin Res Cardiol 2018;107:287-303.

6. Rathod KS, Koganti S, Iqbal MB, et al. Contemporary trends in cardiogenic shock: Incidence, intra-aortic balloon pump utilisation and outcomes from the London Heart Attack Group. Eur Heart J Acute Cardiovasc Care 2018;7:16-27.

7. Thiele H, Zeymer U, Thelemann N, et al. Intraaortic Balloon Pump in Cardiogenic Shock Complicating Acute Myocardial Infarction: Long-Term 6-Year Outcome of the Randomized IABP-SHOCK II Trial. Circulation 2018;139:395-403.

8. Hochman JS, Sleeper LA, Webb JG, et al. Early revascularization and long-term survival in cardiogenic shock complicating acute myocardial infarction. JAMA 2006;295:2511-5.

9. Perera D, Stables R, Clayton T, et al. Long-term mortality data from the balloon pump-assisted coronary intervention study (BCIS-1): a randomized, controlled trial of elective balloon counterpulsation during high-risk percutaneous coronary intervention. Circulation 2013;127:207-12.

Cite this article as: Saia F. Shifting the attention from devices to treatment: the lesson from IABP-SHOCK II and other trials in cardiogenic shock. J Thorac Dis 2019;11(11):E206-E209. doi: 10.21037/jtd.2019.10.66
10. Seyfarth M, Sibbing D, Bauer I, et al. A randomized clinical trial to evaluate the safety and efficacy of a percutaneous left ventricular assist device versus intraaortic balloon pumping for treatment of cardiogenic shock caused by myocardial infarction. J Am Coll Cardiol 2008;52:1584-8.

11. Ouweneel DM, Eriksen E, Sjauw KD, et al. Percutaneous Mechanical Circulatory Support Versus Intra-Aortic Balloon Pump in Cardiogenic Shock After Acute Myocardial Infarction. J Am Coll Cardiol 2017;69:278-87.

12. Schrage B, Ibrahim K, Loehn T, et al. Impella Support for Acute Myocardial Infarction Complicated by Cardiogenic Shock. Circulation 2019;139:1249-58.

13. Alushi B, Douedari A, Froehlig G, et al. Impella versus IABP in acute myocardial infarction complicated by cardiogenic shock. Open Heart 2019;6:e000987.

14. Basir MB, Kapur NK, Patel K, et al. Improved Outcomes Associated with the use of Shock Protocols: Updates from the National Cardiogenic Shock Initiative. Catheter Cardiovasc Interv 2019;93:1173-83.

15. Evidence-based medicine. A new approach to teaching the practice of medicine. JAMA 1992;268:2420-5.

16. Baran DA, Grines CL, Bailey S, et al. SCAI clinical expert consensus statement on the classification of cardiogenic shock: This document was endorsed by the American College of Cardiology (ACC), the American Heart Association (AHA), the Society of Critical Care Medicine (SCCM), and the Society of Thoracic Surgeons (STS) in April 2019. Catheter Cardiovasc Interv 2019;94:29-37. 